\title{
Recomendación del CAVEI de vacunación antineumocócica en adultos
}

\section{CAVEI recommendation for pneumococcal vaccine use in adults}

\author{
Mario Calvo ${ }^{1,2}$, Jaime Inostroza1,3, Magdalena Bastías1, Jeannette Dabanch¹,4, Jaime Cerda1,5, Cecilia González,6, \\ Adiela Saldaña 1,7, Eduardo Díaz ${ }^{1,8}$, María Luz Endeiza1,9, Jaime Rodríguez ${ }^{1,10}$, Solange Santillana ${ }^{1,11}$ y Johanna Acevedo ${ }^{1,12}$
}

\author{
${ }^{1}$ Comité Asesor de Vacunas y Estrategias de Inmunización, Ministerio de Salud de Chile. \\ ${ }^{2}$ Instituto de Medicina, Facultad de Medicina, Universidad Austral de Chile. \\ ${ }^{3}$ Facultad de Medicina, Universidad de La Frontera, Chile. \\ ${ }^{4}$ Hospital Clínico Universidad de Chile, Universidad de Valparaíso, Chile. \\ ${ }^{5}$ Departamento de Salud Pública, Facultad de Medicina, Pontificia Universidad Católica de Chile. \\ ${ }^{6}$ Departamento de Inmunizaciones, Ministerio de Salud de Chile. \\ 7 Instituto de Salud Pública de Chile. \\ ${ }^{8}$ Facultad de Medicina, Universidad de Chile. \\ ${ }^{9}$ Clínica Universidad de los Andes, Chile. \\ ${ }^{10}$ Departamento de Infectología, Clínica Alemana de Santiago, Chile. \\ ${ }^{11}$ SEREMI de Salud Región de Valparaíso, Chile. \\ ${ }^{12}$ Departamento de Epidemiología, Ministerio de Salud de Chile.
}

\section{Introducción}

$\mathrm{L}$ a vacunación antineumocócica se ha realizado desde principios del siglo pasado, es decir, antes del inicio de los antimicrobianos, con células bacterianas completas o polisacáridos capsulares. Siendo una causa importante de morbimortalidad, el interés por las inmunizaciones contra Streptococcus pneumoniae ha sido permanente en diferentes países; no obstante, las decisiones son muy variadas de país a país. Por ejemplo, Estados Unidos de América (E.U.A.) recomienda una vacunación secuencial con vacuna conjugada 13 valente (PCV13) seguida de vacuna polisacárida 23 valente (PSV23) para la protección de los adultos mayores ${ }^{1}$, mientras que en Europa los esquemas son, en general, más simples. De 31 países europeos, en cinco se utiliza la estrategia combinada de PCV13 seguida de PSV23, en cinco se utiliza sólo PCV13, en 11 se recomienda PSV23 y en 10 no hay recomendación de vacuna antineumocócica ${ }^{2}$. En este contexto, se decidió realizar una recomendación para Chile basada en su epidemiología local.

La infección neumocócica invasora es una enfermedad grave y de alta mortalidad. En Chile, entre los años 2007 y 2017 se reportaron al Instituto de Salud Pública (ISP) 1.893 cepas de S. pneumoniae participando en infección invasora en pacientes mayores de 65 años (de un total de 8.400 cepas del período). No obstante, esto es una minoría de los casos de infección neumocócica en este grupo etario, ya que $90 \%$ de los casos de infección invasora corresponde a neumonía bacteriémica, siendo poco frecuente el aislamiento bacteriano en estos casos.

La neumonía es una enfermedad frecuentemente causada por S. pneumoniae. En Chile, las estadísticas de mortalidad global por neumonía (Códigos CIE-10 J12$\mathrm{J} 16, \mathrm{~J} 18$ ) dan cuenta en el año 2015 de 3,9\% de las causas de muerte sobre los 65 años $^{3}$ y de $3,7 \%$ de las causas de muerte sobre los 65 años en el $2016^{4}$.

\section{Situación de la vacunación contra Streptococcus pneumoniae en Chile}

La vacuna PSV23 fue introducida al Programa Nacional de Inmunizaciones en el año 2007 para adultos mayores de 65 años, a pesar de que los datos de efectividad son controversiales. Como se ha mencionado, esto ha 


\begin{tabular}{|cc|}
\hline $\begin{array}{c}\text { Tabla 1. Cobertura de vacunación antineumocócica PCV13 } \\
\text { o PSV23 en el adulto de } 65 \text { años en Chile, 2012-2017 }\end{array}$ \\
\hline Cohorte & Cobertura \\
1947 & 60,5 \\
1948 & 59,7 \\
1949 & 53,8 \\
1950 & 46,8 \\
1951 & 39,0 \\
1952 & 30,8 \\
\hline $\begin{array}{l}\text { Fuente: Departamento de Inmunizaciones, Ministerio de Salud } \\
\text { de Chile. }\end{array}$ \\
\hline
\end{tabular}

llevado a que tengamos realidades como la de E.U.A., en que se busca aumentar la efectividad administrando primeramente la PCV13 y luego PSV23 a este grupo etario, lo que contrasta con países europeos como Francia y Países Bajos, donde no es administrada de rutina. Como ya se ha señalado, otros países en Europa vacunan sólo con PCV13 o sólo con PSV23.

Al revisar los datos provistos por el ISP, se ve que $60,5 \%$, de la cohorte nacida en 1947 ha recibido PCV13 o PSV23, siendo la cohorte más vacunada, cifra que disminuye hasta llegar a la cohorte de 1952, en que sólo $30,8 \%$ del grupo está vacunado (Tabla 1). Este descenso en las siguientes cohortes se explica porque hay personas que son vacunadas en los años siguientes al que cumplen 65 años; el registro nacional de vacunas permite que se detecte que no han recibido la vacuna en el momento programado $\mathrm{y}$, en consecuencia, sea administrada en forma posterior.

\section{Efectividad de la inmunización}

Los dos tipos de vacunas disponibles en Chile han sido estudiados en otras latitudes. Por ser los últimos resúmenes hallados en la literatura científica al respecto, nos referimos a las revisiones sistemáticas de Moberley et al. ${ }^{5}$, Ewald et al. ${ }^{6}$ y de Falkenhorst et al. ${ }^{7}$.

La revisión sistemática de Moberley separó las distintas poblaciones y resultados, y analizó sólo PSV23. En los ensayos controlados aleatorizados para el resultado "enfermedad neumocócica invasora" se demuestra una efectividad de $74 \%$, siendo en los adultos sanos de bajos ingresos $86 \%$, para los sanos de altos ingresos $80 \%$ y para los adultos con enfermedad crónica no se encontró diferencias. Para el resultado de "neumonía por todas las causas", la efectividad fue de $28 \%$, siendo de $46 \%$ en los países de bajos ingresos, mientras en los otros dos grupos las diferencias no fueron estadísticamente significativas. En el caso de prevenir la neumonía neumocócica, la efectividad fue de $74 \%$.

La revisión sistemática de Ewald et al. evaluó el uso de vacunas conjugadas habiendo incluido sólo cinco estudios en personas sobre 60 años de edad. No mostró diferencias en mortalidad, pero sí en la prevención de la enfermedad neumocócica invasora, concluyendo un OR de 0,43 ( $\left.\mathrm{IC}_{95 \%} 0,36-0,51\right)$, en general. Asimismo, hubo una reducción de neumonía por todas las causas con un OR 0,93 ( IC $\left._{95 \%} 0,88-0,97\right)$.

El trabajo de Falkenhorst et al. evaluó el uso de PSV23 en mayores de 60 años, mostrando una disminución de $73 \%$ en la enfermedad neumocócica invasora en ensayos clínicos, $45 \%$ en los estudios de cohortes y $59 \%$ en los estudios de casos y controles. La disminución de la neumonía neumocócica de cualquier serotipo fue $64 \%$ en los ensayos clínicos y $48 \%$ en las cohortes. La mayor efectividad en seguimientos más cortos plantea la posibilidad de la pérdida de inmunidad.

Los estudios no han mostrado impacto en mortalidad general.

La comparación en adultos entre ambas vacunas se ha hecho mediante medición de actividad opsonofagocítica antineumocócica, mostrándose superior para PCV13 que para PSV23 ${ }^{8}$. Similares resultados mostró el trabajo de Shiramoto et al. ${ }^{9}$, trabajo que constató, además, mayores reacciones adversas locales para PCV13, pero no graves.

\section{Análisis de los aislados de Streptococcus pneumoniae por el ISP en Chile (2007-2017)}

Al revisar las cepas aisladas en los últimos 11 años en Chile* para toda la población, se observa que en el período 2007 a 2010 , entre 62,8 y 79,7\% de las cepas aisladas cada año estaban incluidas dentro de las cubiertas por PCV13, siendo estas cifras entre 67,9 y $84,6 \%$ para PSV23. Desde entonces, se aprecia un descenso, tal que el año 2017 48,2\% de las cepas aisladas están incluidas en PCV13 y $68,0 \%$ en PSV23. Este fenómeno podría tratarse de una sustitución como consecuencia de la misma vacunación (Tabla 2).

Si consideramos para el período total 2007-2017 en los pacientes mayores de 65 años, de 1.893 cepas, 1.370 $(72,3 \%)$ estaban incluidas en la vacuna PSV23 y 1.085 $(57,3 \%)$ en la PCV13 (Tabla 3, Figura 1). La diferencia es $15,1 \%$. Esto significa que de vacunar con una $u$ otra, la PCV13 debiese ser un $26 \%$ más activa en ese resultado para equiparar la eficacia.

* Información obtenida por Ley de Transparencia, solicitudes al ISP códigos AO005T0002495 y AO005T0002653. 
Para el mismo período, en los pacientes de 60 a 64 años, de 556 cepas, $431(77,5 \%)$ estaban incluidas en la vacuna PSV23 y 319 (57,3\%) en la PCV13. La diferencia es $20,2 \%$. Esto significa que de vacunar con una u otra, la PCV13 debiese ser 35\% más activa en las cepas que cubre para equiparar la eficacia.

\section{De lo revisado, se concluye que:}

- Para ser equivalente en resultado clínico, la efectividad sobre las cepas que cubren ambas vacunas debería ser para la vacuna conjugada 13 valente, de alrededor de $30 \%$ mayor, lo que según los resultados del estudio CAPITA es difícil que se presente, haciendo difícil pensar que pueda dar resultados significativamente superiores a la PSV23 ${ }^{10}$.

- La cantidad de muestras provenientes de procesos invasores, a las que ofrece cobertura, PSV23 es levemente superior a la que da PCV13, y esta diferencia parece aumentar en los últimos años. Para las cepas aisladas el año 2017, 48,2\% correspondió a serotipos incluidos en la vacuna PCV13, en cambio esta cifra es $68,0 \%$ para la vacuna PSV23.

- La estrategia de vacunación secuencial no tiene actualmente validado un aumento de su efectividad clínica en la población general.

- Las tasas de vacunación son sub-óptimas.

EI CAVEI considera importante mantener la vacuna PSV23 en el Programa Nacional de Inmunizaciones para el adulto mayor de 65 años. No obstante, es urgente tomar medidas que aseguren la adherencia de esta población a la vacunación, tal de repercutir en los resultados clínicos de este grupo etario.

Nota: esta recomendación reemplaza la recomendación del CAVEI de vacunación antineumocócica en adultos del 26 de septiembre de 2018.

Comité Asesor en Vacunas y Estrategias de Inmunización

Santiago, 26 de noviembre de 2019.
Tabla 2. Cepas aisladas de Streptococcus pneumoniae y cobertura según vacuna antineumocócica, Chile 2007-2017

\begin{tabular}{|c|c|c|c|c|c|}
\hline \multirow{2}{*}{$\begin{array}{l}\text { Año } \\
2007\end{array}$} & \multirow{2}{*}{$\begin{array}{c}\text { Cepas } \\
\text { aisladas } \\
887\end{array}$} & \multicolumn{2}{|c|}{$\begin{array}{c}\text { Incluidas en PCV13 } \\
\text { n }(\%)\end{array}$} & \multicolumn{2}{|c|}{$\begin{array}{c}\text { Incluidas en PSV23 } \\
\text { n } \quad(\%)\end{array}$} \\
\hline & & 594 & $(66,9)$ & 668 & $(75,3)$ \\
\hline 2008 & 870 & 663 & $(76,2)$ & 725 & $(83,3)$ \\
\hline 2009 & 901 & 566 & $(62,8)$ & 612 & $(67,9)$ \\
\hline 2010 & 775 & 618 & $(79,7)$ & 656 & $(84,6)$ \\
\hline 2011 & 725 & 531 & $(73,2)$ & 580 & $(80,0)$ \\
\hline 2012 & 671 & 437 & $(65,1)$ & 538 & $(80,1)$ \\
\hline 2013 & 695 & 410 & $(58,9)$ & 505 & $(72,6)$ \\
\hline 2014 & 661 & 369 & $(55,8)$ & 450 & $(68,0)$ \\
\hline 2015 & 670 & 366 & $(54,6)$ & 501 & $(74,7)$ \\
\hline 2016 & 770 & 384 & $(49,8)$ & 537 & $(69,7)$ \\
\hline 2017 & 775 & 374 & $(48,2)$ & 527 & $(68,0)$ \\
\hline Total & 8.400 & 5.312 & $(63,2)$ & 6.299 & $(74,9)$ \\
\hline
\end{tabular}

Tabla 3. Cobertura total de serotipos de Streptococcus pneumoniae según vacuna y grupo etario, Chile 2007-2017

$<12$ meses 12 a 59 meses 5 a 64 años $>65$ años

$\begin{array}{lllll}\text { PCV-13 } & 72,8 \% & 78,3 \% & 62,9 \% & 57,3 \% \\ \text { PSV-23 } & 77,8 \% & 78,3 \% & 79,7 \% & 72,3 \% \\ \text { PSV-23 } & & & 87,6 \% & 82,9 \%\end{array}$

PSV-23

$87,6 \% \quad 82,9 \%$

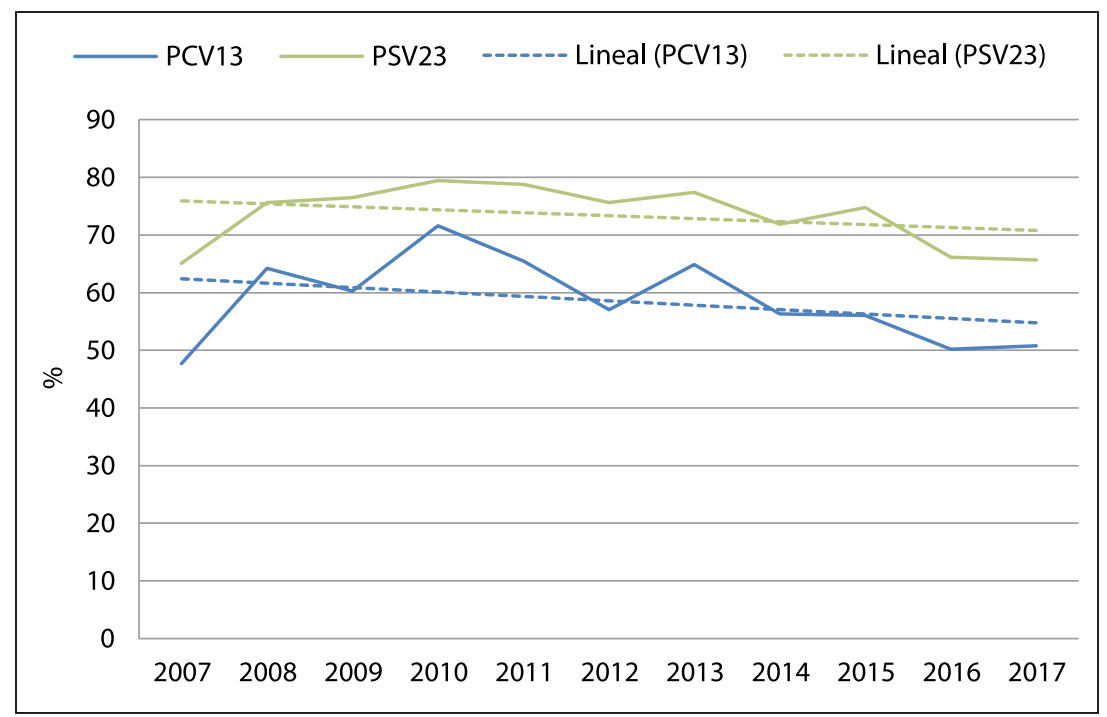

Figura 1. Porcentaje de cobertura PCV13 y PSV13 en personas mayores de 65 años, Chile 2007-2017. 


\section{Referencias bibliográficas}

1.- United States Centers for Disease Control and Prevention. Recommended adult immunization schedule for ages 19 years or older, United States, 2019 [Internet]. 2019. Available from: https://www.cdc.gov/vaccines/schedules/hcp/ imz/adult.html.

2.- European Centre for Disease Prevention and Control. Pneumococcal Disease: Recommended vaccinations [Internet]. 2019. Available from: https://vaccine-schedule.ecdc.europa.eu/ Scheduler/ByDisease?SelectedDiseaseId $=25 \& S$ electedCountryIdByDisease $=-1$.

3.- Instituto Nacional de Estadísticas-Chile. Anuario de Estadísticas Vitales 2015 [Internet]. 2017. Available from: https://www.ine.cl/docs/ default-source/publicaciones/2017/síntesis-deestadísticas-vitales-2015.pdf?sfvrsn=7.

4.- Instituto Nacional de Estadísticas-Chile. Estadísticas Vitales Informe Anual 2016 [Internet]. 2018. Available from: https://www. ine.cl/docs/default-source/demográficas-y- vitales/vitales/anuarios/2016/vitales-2016. pdf?sfvrsn $=15$.

5.- Moberley S, Holden J, Tatham DP, Andrews RM. Vaccines for preventing pneumococcal infection in adults. Cochrane Database Syst Rev 2013; 2013 (1). CD000422. doi: 10.1002/14651858.

6.- Ewald H, Briel M, Vuichard D, Kreutle V, Zhydkov A, Gloy V. The clinical effectiveness of pneumococcal conjugate vaccines: a systematic review and meta-analysis of randomized controlled trials. Dtsch Arztebl Int 2016; 113 (9): 139-46. doi: 10.3238/ arztebl.2016.0139.

7.- Falkenhorst G, Remschmidt C, Harder T, Hummers-Pradier E, Wichmann O, Bogdan C. Effectiveness of the 23-valent pneumococcal polysaccharide vaccine (PPV23) against pneumococcal disease in the elderly: systematic review and meta-analysis. PLoS One 2017; 12 (1): e0169368. doi: 10.1371/journal. pone. 0169368 .

8.- Jackson LA, Gurtman A, van Cleeff M,
Frenck RW, Treanor J, Jansen KU, et al. Influence of initial vaccination with 13-valent pneumococcal conjugate vaccine or 23-valent pneumococcal polysaccharide vaccine on anti-pneumococcal responses following subsequent pneumococcal vaccination in adults 50 years and older. Vaccine 2013; 31 (35): 3594-602. doi: 10.1016/j.vaccine.2013.04.084.

9.- Shiramoto M, Hanada R, Juergens C, Shoji Y, Yoshida M, Ballan B, et al. Immunogenicity and safety of the 13-valent pneumococcal conjugate vaccine compared to the 23 -valent pneumococcal polysaccharide vaccine in elderly Japanese adults. Hum Vaccin Immunother [Internet]. 2015; 11 (9): 2198-206. Available from: http://www.tandfonline.com/ doi/full/10.1080/21645515.2015.1030550.

10.- Bonten M, Huijits S, Bolkenbaas M, Webber C, Patterson S, Gault S, et al. Polysaccharide conjugate vaccine against pneumococcal pneumonia in adults. N Engl J Med 2015; 372 (12): 1114-25. doi: 10.1056/NEJMoa1408544. 